Infinite Dimensional Analysis, Quantum Probability

and Related Topics

Vol. 13, No. 4 (2010) 691-692

(C) World Scientific Publishing Company

DOI: $10.1142 / \mathrm{S} 0219025710004255$

\title{
AUTHOR INDEX (Vol. 13)
}

Accardi, L., Ohno, H. \& Mukhamedov, F., Quantum Markov fields on graphs

Accardi, L., Ji, U. C. \& Saitô, K., Exotic Laplacians and associated stochastic processes (Errata)

Accardi, L., Barhoumi, A. \& Riahi, A., White noise Lévy-Meixner processes through a transfer principal from one-mode to onemode type interacting Fock spaces

Accardi, L., Ouerdiane, H. \& Rebeï, H., On the quadratic Heisenberg group

Ayupov, A. Sh. \& Kudaybergenov, K. K., Derivations on algebras of measurable operators

Barhoumi, A., see Accardi

Bhat, B. V. R. \& Mukherjee, M., Inclusion systems and amalgamated products of product systems

Butko, Y. A., Grothaus, M. \& Smolyanov, O. G., Lagrangian Feynman formulas for second-order parabolic equations in bounded and unbounded domains

Dey, S., Liftings of covariant representations of $W^{*}$ correspondences

Dhahri, A., Fagnola, F. \& Rebolledo, R., The decoherence-free subalgebra of a quantum Markov semigroup with unbounded generator

Fagnola, F., see Dhahri
Fichtner, K.-H., Inoue, K. \& Ohya, M., On the low13 (2010) 165 temperature behavior of the infinite-volume ideal Bose gas

Fidaleo, F. \& Mukhamedov,

13 (2010) $345 \quad$ F., Ergodic properties of Bogoliubov automorphisms in free probability

Grothaus, M., see Butko

Hasebe, T., Monotone convolution and monotone infinite divisibility from complex analytic viewpoint

13 (2010) 435

13 (2010) 551

13 (2010) 305

13 (2010) 435

13 (2010) 1

13 (2010) 377

13 (2010) 511

13 (2010) 413

13 (2010) 413
Hasebe, T., White noise analysis on manifolds and the energy representation of a gauge group

He, H., Fleming-Viot processes in an environment

Hiroshi, A., On the local structure of the representation of a local gauge group

Hong, W. \& Zhang, L., Branching structure for the transient $(1, R)$-random walk in random environment and its applications

Inoue, K., see Fichtner

Ji, U. C., see Accardi

Konsowa, M. H., Random walks and dimensions of random trees

Kubo, I. \& Kuo, H.-H., MRMfactors for the probability measures in the Meixner class

Kudaybergenov, K. K., see Ayupov

Kula, A. \& Wysoczański, J., Noncommutative Brownian motions indexed by partially ordered sets
13 (2010) 39

13 (2010) 393

13 (2010) 377

13 (2010) 111

13 (2010) 619

13 (2010) 489

13 (2010) 223

13 (2010) 589

13 (2010) 39

13 (2010) 345

13 (2010) 677

13 (2010) 525

13 (2010) 305

13 (2010) 629 
Kuo, H.-H., see Kubo

Lee, Y.-J. \& Shih, H.-H., An application of the SegalBargmann transform to the characterization of Lévy white noise measures

Liu, W., Invariance of subspaces under the solution flow of SPDE

Luo, D., Well-posedness of Fokker-Planck type equations on the Wiener space

Marinelli, C. \& Röckner, M., On uniqueness of mild solutions for dissipative stochastic evolution equations

Mouayn, Z. \& Touhami, A., Probability distributions attached to generalized Bargmann-Fock spaces in the complex plane

Mukhamedov, F., see Accardi

Mukhamedov, F., see Fidaleo

Mukherjee, M., see Bhat

Ohno, H., see Accardi

Ohya, M., see Fichtner

Ouerdiane, H., see Accardi

Prévôt, C. I., Existence, uniqueness and regularity w.r.t. the initial condition of mild solutions of SPDEs driven by Poisson noise

Privault, N., Random Hermite polynomials and Girsanov identities on the Wiener space

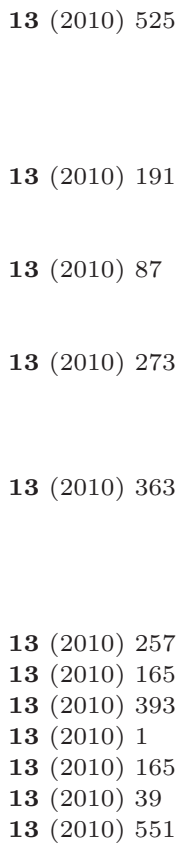

13 (2010) 525

13 (2010) 191

13 (2010) 87

13 (2010) 273

13 (2010) 363

13 (2010) 257

13 (2010) 165

13 (2010) 393

13 (2010) 1

13 (2010) 165

13 (2010) 39

13 (2010) 551

13 (2010) 133

13 (2010) 663
Rebeï, H., see Accardi

Rebolledo, R., see Dhahri

Riahi, A., see Accardi

Röckner, M. \& Wang, F.-Y., Log-Harnack inequality for stochastic differential equations in Hilbert spaces and its consequences

Röckner, M., see Marinelli

Saigo, H., A simple proof for monotone CLT

Saitô, K., see Accardi

Sakbaev, V. Zh., Stochastic properties of degenerated quantum systems

Shih, H.-H., see Lee

$\mathrm{Si}, \mathrm{Si}$, Multiple Markov generalized Gaussian processes and their dualities

Smolyanov, O. G., see Butko

Stan, A. I., Best constants in norms of retarded Wick products

Touhami, A., see Mouayn

Wang, F.-Y., see Röckner

Wysoczański, J., bm-Independence and bm-central limit theorems associated with symmetric cones

Wysoczański, J., see Kula

Yoshida, H., Remarks on the free relative entropy and the free Fisher information distance

Zhang, L., see Hong
13 (2010) 551

13 (2010) 413

13 (2010) 435

13 (2010) 27

13 (2010) 363

13 (2010) 339

13 (2010) 345

13 (2010) 65

13 (2010) 191

13 (2010) 99

13 (2010) 377

13 (2010) 347

13 (2010) 257

13 (2010) 27

13 (2010) 461

13 (2010) 629

13 (2010) 243

13 (2010) 589 